
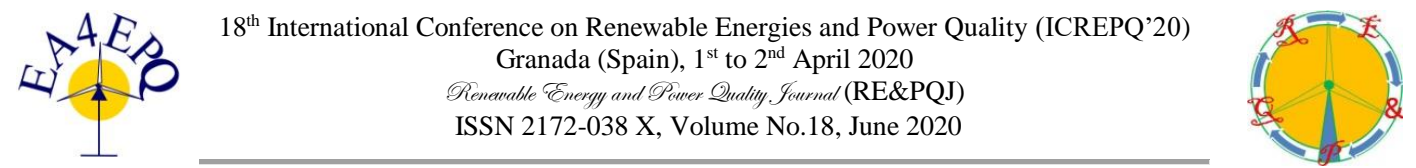

\title{
Reconfigurable Droop-Based DC Microgrids
}

\author{
Abdelsalam A. Eajal ${ }^{1}$, Aboelsood Zidan ${ }^{2}$, Ehab F. El-Saadany ${ }^{3}$, Magdy Salama $^{4}$, and Hatem Zeineldin ${ }^{5}$ \\ ${ }^{1,4}$ Department of Electrical Engineering, University of Waterloo, Waterloo, Canada \\ ${ }^{3,5}$ Advanced Power and Energy Centre, EECS Department, Khalifa University, Abu Dhabi
}

Emails: ${ }^{1}$ abdel.eajal@uwaterloo.ca, ${ }^{2}$ aboelsoodzidan@yahoo.com, ${ }^{3}$ ehab.elsadaany@ku.ac.ae, 고.salama@uwaterloo.ca, ${ }^{5}$ hatem.zeineldin@ku.ac.ae

\begin{abstract}
This paper presents a mathematical formulation for loss minimization of reconfigurable and droop-based dc microgrids (dc MGs) during islanding. The objective is achieved by adjusting the droop settings of the distributed generators (DGs) and reconfiguring the topology of the islanded MG. The reconfiguration problem is formulated as a mixed-integer nonlinear optimization problem and solved via a metaheuristic technique: genetic algorithms (GAs). The proposed formulation takes into account: 1) the unavailability of a slack bus; 2) the droop controllability of DGs. The proposed model is tested on a six-bus islanded dc MG. The case studies demonstrate the effectiveness of the developed formulation in jointly optimizing the droop characteristics and MG topology for minimizing the power losses, and by product; improving the overall voltage profile.
\end{abstract}

Key words. DC microgrids, distributed generation, droop control, and reconfiguration.

\section{Introduction}

The concept of a grid of multi microgrids with islanding capability is proposed towards enabling a more reliable, secure, and automated power grid [1]. In addition, the advances in dc technologies such as renewables, modern $\mathrm{dc}$ loads, and dc circuit breakers put forward the realization of reconfigurable dc microgrids (MGs) [2]. An example of a reconfigurable dc MG is depicted in Figure 1. The dc breakers allow each dc MG to reconfigure its own topology to adapt to any islanding conditions [3]. Each islanded dc MG can disconnect from the hosting ac grid and independently supply its own demand though its local distributed generators (DG). To cope with the islanding and the lack of a slack bus, the DGs adopt droop characteristics to control the voltage and share the load proportionally to their ratings [4]. However, without proper settings, the droop characteristics can result in high power losses. This paper proposes a joint optimization of the droop parameters and MG topology so that the power losses are minimum during islanding.

Similar to distribution systems, MGs are typically operated in a radial structure for the ease of protection coordination and operation [4]. Radial topology is also an operational practice for islanded systems.

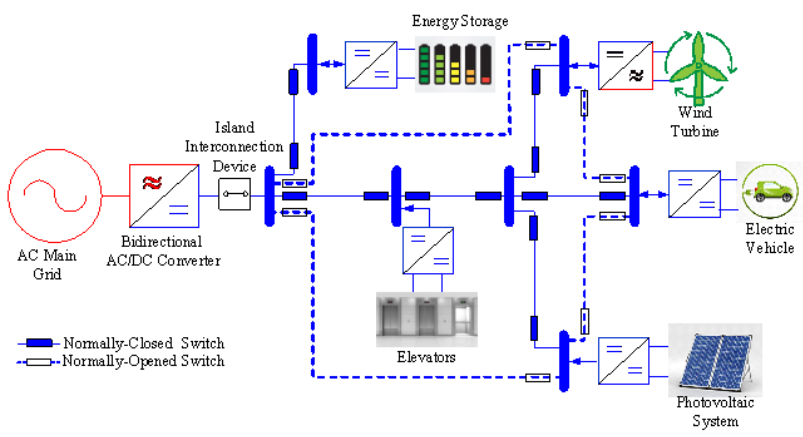

Figure 1 An example of a reconfigurable dc microgrid layout.

The problem of identifying a radial topology for conventional distribution networks is a well-documented subject due to its combinatorial nature. The reader is referred to [5] for an up-to-date review. Several solution methods have been proposed for the combinatorial reconfiguration problem: deterministic methods such as mixed-integer linear programming (MILP) [6], [7], and heuristic methods such as genetic algorithm (GA) [8], particle swarm optimization (PSO) [9], and ant colony optimization (ACO) [10]. Some decomposition techniques have been ALSO proposed such as the Bender's decomposition [11]. In addition to decomposition, a convex relaxation of the reconfiguration problem has been recently addressed in [12], [13], [14], [15]. The reconfiguration problem for MGs is quite different because of the unique characteristics of MGs such as the islanding capability, the absence of a slack bus during the islanding period, and the implementation of droop control in which the frequency and voltage is drooped with load increase. Thus, the reconfiguration problem for droop-controlled islanded MGs has been the focus of a recent work [8], but only for ac type networks. The reconfiguration problem for islanded droop-based dc MGs is investigated in this paper. The paper introduces a mathematical formulation for join network configuration and DG droop parameter optimization. The objective of the optimization problem is taken as the minimization of total power loss during islanding.

In this paper, Section 2 discusses droop-controlled DGs. Section 3 presents the proposed formulation. Section 4 
reports the case studies with discussions. Section 5 offers conclusions.

\section{Droop Control of Distrusted Generation}

In droop control, the terminal voltage of the dc-type DG units are regulated autonomously based on local measurements of the DG active power output, as indicated by (1) and Figure 2.

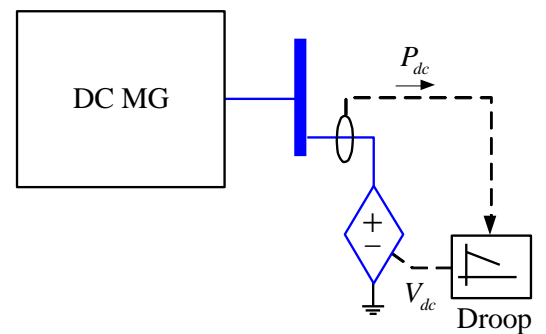

Figure 2 Droop-controlled DG model

$$
V_{d c}=V_{d c}^{*}-m_{p} P_{G, d c}
$$

where $m_{p}$ is the active power static droop gains of the DG unit and $V_{d c}^{*}$ is the DG reference or no-load voltage. The droop equation (1) shows the dc voltage is directly coupled with the active power. It should be mentioned that the active power sharing among droop-controlled DGs is no exact because of different voltage drops across the lines. Power sharing is out of the scope of the present work. Because of unequal voltage drop at the DGs terminals, some DGs might become overloaded while other DGs are under-utilized. If any DG unit reaches its rating, it is then switched from droop control mode to constant current mode.

\section{Problem Formulation}

The reconfiguration problem for islanded droop-controlled dc MGs is formulated as a mixed-integer nonlinear optimization problem and solved by the genetic algorithm (GA) [5]. The developed formulation takes into account the physical law of the power flow, the security constraint of the bus voltage, the thermal capacity of the lines, the ratings of the DGs, and the radial topology of the MG. The proposed mathematical model is described by (2)-(10).

\section{A. Objective Function}

$$
\min P_{\text {Loss }}=\left[V_{d c}\right]_{1 \times n}^{T}\left[G_{d c}\right]_{n \times n}\left[V_{d c}\right]_{n \times 1}
$$

\section{B. Constraints}

1. DC Power Flow Law

$$
P_{G, d c, b}-P_{D, d c, b}=V_{d c, b} \sum_{k=1}^{n} V_{d c, k} G_{d c, b k} U_{s w, b k}
$$

\section{Bus Voltage Security Limits}

$$
\begin{gathered}
V_{d c, \text { min }} \leq V_{d c, b} \leq V_{d c, \max }, \forall b \in \Omega_{\text {Load }} \\
V_{d c, b}^{*}-V_{d c, b} \geq \Delta V_{d c, b}^{\max }, \forall b \in \Omega_{G}
\end{gathered}
$$

3. Line Currying Capacity Limits

$$
\left|I_{d c, b k}\right| \leq I_{d c, b k}^{\max }, \forall b, k \in \Omega_{B}
$$

\section{Droop-Based DG Capacity Limits}

$$
P_{G, d c, b}^{\min } \leq P_{G, d c, b} \leq P_{G, d c, b}^{\max }
$$

\section{Droop Parameters Limits}

$$
\begin{gathered}
D_{d c, b}^{\min } \leq D_{d c, b} \leq D_{d c, b}^{\max } \\
\text { where } D_{d c, b}=\left[m_{p, b}, V_{d c, b}^{*}\right]^{T}, \forall b \in \Omega_{G}
\end{gathered}
$$

\section{Radial Topology Requirement}

$$
\begin{gathered}
\sum_{b, k \in \Omega_{B}} U_{s w, b k}=n-1 \\
U_{s w, b k}= \begin{cases}1 & \text { if line } b k \text { is on } \\
0 & \text { if line } b k \text { is off }\end{cases}
\end{gathered}
$$

The objective functions in (2) is set to loss minimization. Nevertheless, the objective function can always be extended to include other operational practices such as the minimization of switching operations. As (2) suggests, the power loss is a function of the bus voltages and the dc network topology which is described by the bus conductance matrix $\left[G_{d c}\right]$. The physical law of the power flow at each bus $\forall b \in \Omega_{B}$ is preserved by setting the dc power flow equation shown in (3) as an equality constraint. In order to keep the voltage at load buses in (4) within the operational limits, e.g., $5 \%$, the voltage at the DG terminals are restricted to $2.5 \%$ of the nominal voltage, as described by (5). The carrying capacity $I_{d c, b k}^{\max }$ of line $b k$ is set as a technical constraint, as expressed in (6) to avoid line overloading and any possible consequences. The DG units also impose some technical constraints represented in their ratings $P_{G, d c}^{\max }$, as shown in (7). The DG model can be extended to include the DG unit availability. It should be noted that in case of renewables, the upper bound on DG output power is defined by the minimum of the power available from the renewable energy source and the rated capacity of the interfacing dc/dc converter. Droop parameters also shall be varied within a certain range as described by (8). In practice, the limits on the droop parameters should be defined based on a stability criteria.

Imposing radiality in the reconfiguration problem is not trivial as described by (9) and (10). Hence, several attempts have been made in this regard such as [16], [11], [17], [18]. In this work, heuristic rules are followed to exclude all possible configurations that result in a nonradial topology and isolated buses [19]:

1) The dc MG is represented as a tree with $n$ nodes and $n-1$ branches.

2) All switches corresponding to leaf nodes are switched on to avoid load isolation. 
3) Only one switch from a vector of branches that are common between two fundamental loops can be opened.

4) At least one switch from a vector of non-common branches must be switched on to prevent islanding of exterior nodes.

5) The switches corresponding to branches that are connected to the same interior node cannot be opened simultaneously.

Once the radiality check is passed, the other constraints in (3)-(8) can be checked.

\section{Test System and Case Studies}

The proposed model was implemented in MATLAB $^{\odot}$ computing environment and tested on a six-bus islanded dc MG. As depicted in the one line diagram shown in Figure 3 , the de network involves two tie lines, TL1 and TL2, allowing for adaptive reconfiguration of the $\mathrm{dc} M G$ as the operating mode changes. The dc feeders have been designed based on a voltage drop criteria [20]. The line specifications are listed in TABLE I. The test dc MG involves three DGs with a total capacity of $45 \mathrm{~kW}$ and are all equipped with droop characteristics for the islanded mode of operation. The DG ratings and default droop paramours are tabulated in TABLE II. The DG location and size are assumed to be determined at the planning stage. The three DGs supply a total load of $42 \mathrm{~kW}$, leaving only $6.667 \mathrm{~kW}$ as an available reserve capacity. The base $\mathrm{kW}$ and dc voltage were taken as $10 \mathrm{~kW}$ and $600 \mathrm{~V}$, respectively.

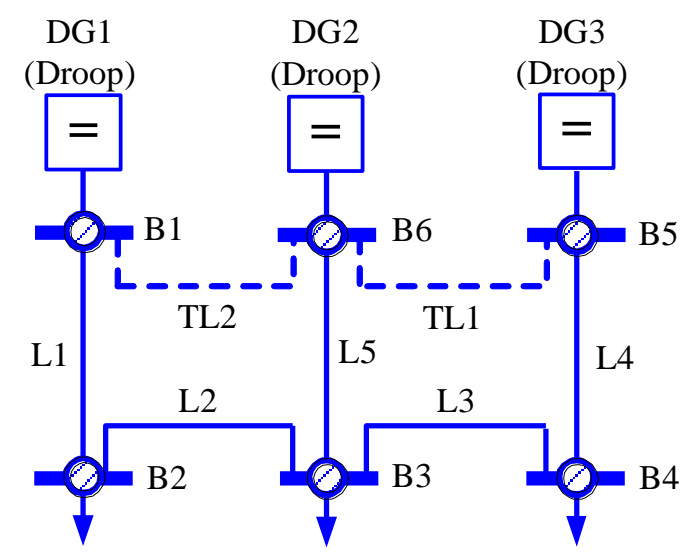

Figure 3 One line diagram of a six-bus dc microgrid.

\begin{tabular}{|c|c|c|c|c|c|c|}
\hline Line & From & To & $\begin{array}{c}\text { Length } \\
(\mathrm{km})\end{array}$ & $\begin{array}{l}\text { Current } \\
\text { Capacity } \\
\text { (A) }\end{array}$ & $\begin{array}{c}R \\
(\Omega)\end{array}$ & $\begin{array}{l}X / R \\
\text { Ratio }\end{array}$ \\
\hline L1 & 1 & 2 & 0.8 & 33.33 & 0.395 & 0.18 \\
\hline L2 & 2 & 3 & 0.5 & 16.67 & 0.735 & 0.06 \\
\hline L3 & 3 & 4 & 0.3 & 16.67 & 0.699 & 0.04 \\
\hline L4 & 4 & 5 & 0.1 & 25.00 & 0.588 & 0.02 \\
\hline L5 & 3 & 6 & 0.7 & 16.67 & 0.649 & 0.10 \\
\hline TL1 & 5 & 6 & 0.4 & 16.67 & 0.588 & 0.06 \\
\hline TL2 & 6 & 1 & 0.3 & 16.67 & 0.699 & 0.04 \\
\hline
\end{tabular}

TABLE II BUS DATA OF THE SIX-BUS DC MICROGRID

\begin{tabular}{|c|c|c|c|c|}
\hline Bus & $\begin{array}{c}\text { Load } \\
(\mathrm{kW})\end{array}$ & $\begin{array}{c}\text { DG Rating } \\
(\mathrm{kW})\end{array}$ & $\begin{array}{c}\text { Droop Gain } \\
(\mathrm{V} / \mathrm{W})\end{array}$ & $\begin{array}{c}\text { Reference } \\
\text { Voltage }(\mathrm{V})\end{array}$ \\
\hline B1 & --- & 20 & $7.5 \times 10^{-4}$ & 1.0 \\
\hline B2 & 20 & --- & --- & --- \\
\hline B3 & 6 & --- & --- & --- \\
\hline B4 & 16 & --- & --- & --- \\
\hline B5 & --- & 15 & $1.0 \times 10^{-3}$ & 1.0 \\
\hline B6 & --- & 10 & $1.5 \times 10^{-3}$ & 1.0 \\
\hline
\end{tabular}

Three case studies are simulated on the test dc MG.

Case 1: Base case: before reconfiguration and optimization of the droop parameters

Case 2: After reconfiguration but default droop settings

Case 3: Joint reconfiguration and optimal droop settings

Case 1 is the base case in which the network is not reconfigured after islanding and the droop settings of the DG units are not adjusted for the new islanding conditions. The total losses are 0.2204 p.u. for the base configuration (case 1) shown in TABLE III.

Table III The Radial Topology: Case 1-3

\begin{tabular}{|c|c|c|c|c|c|c|c|}
\cline { 2 - 8 } \multicolumn{1}{c|}{} & L1 & L2 & L3 & L4 & L5 & TL1 & TL2 \\
\hline Case 1 & 1 & 1 & 1 & 0 & 1 & 1 & 0 \\
\hline Case 2 & 1 & 1 & 1 & 1 & 1 & 0 & 0 \\
\hline Case 3 & 1 & 1 & 1 & 1 & 1 & 0 & 0 \\
\hline
\end{tabular}

In case 2 , the decision variables are only the status of the switches, $U_{s w, b k} \in\{1,0\}, \forall b, k \in \Omega_{B}$. The total losses are 0.0956 p.u. In case 2 , the MG topology is altered to adapt to the islanding conditions and minimize the power losses. The new radial topology described by the line status is shown in TABLE III. As seen from this table, the line between buses 4 and 5 (line L4) is switched on while the tie line TL1 is opened in order to bring the losses to minimum during islanding.

In case 3, the optimization variables are set as the status of the switches and the droop parameters of each DG, $D_{d c, b}=\left[m_{p, b}, V_{d c, b}^{*}\right]^{T}, \forall b \in \Omega_{G}$. The total losses are 0.0912 p.u. with an optimum configuration the same as case 2 but with adjusting the droop settings of the DGs. The new DG droop settings are shown in TABLE IV. As shown in in this table, the reference voltages of all DGs are set to higher values in order to deliver the required power at lower currents, and thus reducing the resistive losses.

TABLE IV THE OptIMAL Droop SETTINGS: CASE 3
\begin{tabular}{|c|c|c|}
\hline DG & $\begin{array}{c}\text { Droop Gain } \\
\text { (p.u.) }\end{array}$ & $\begin{array}{c}\text { Reference } \\
\text { Voltage (p.u.) }\end{array}$ \\
\hline DG1 & $1.4387 \times 10^{-3}$ & 1.0441 \\
\hline DG2 & $1.1944 \times 10^{-3}$ & 1.0286 \\
\hline DG3 & $2.0094 \times 10^{-3}$ & 1.0289 \\
\hline
\end{tabular}

Joint optimization of the droop parameters and reconfiguration was found to reduce the ohmic losses by approximately $14 \%$. Further, the voltage profile has improved at all buses, as depicted in Figure 4. 


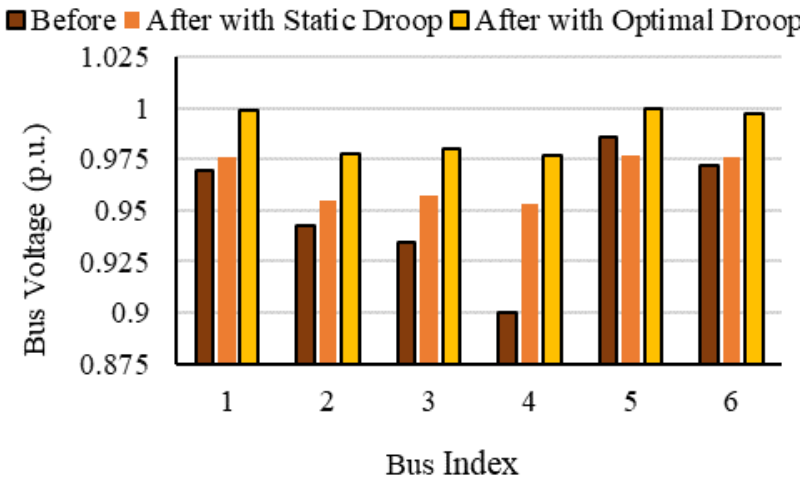

Figure 4 Bus voltages before and after reconfiguration and droop optimization.

\section{Conclusion}

This paper introduced the joint optimization of the dc MG topology and the droop settings of distributed generators when islanding takes a place. The objective was set as loss minimization subject to technical and operational constraints. The test results proved that the operation of an islanded dc MG with reconfiguration capability can be enhanced by simultaneously adjusting the droop settings and reconfiguring the $\mathrm{MG}$ topology. Ongoing work is focusing on reconfiguring dc MGs that are intertied with ac MGs through bidirectional ac/dc converters.

\section{References}

[1] R. H. Lasseter, "Smart distribution: Coupled microgrids," Proc IEEE, vol. 99, (6), pp. 1074-1082, 2011.

[2] T. Dragičević et al, "DC microgrids-Part II: A review of power architectures, applications, and standardization issues," IEEE Transactions on Power Electronics, vol. 31, (5), pp. 3528-3549, 2015.

[3] D. Salomonsson, L. Soder and A. Sannino, "Protection of low-voltage DC microgrids," IEEE Trans. Power Del., vol. 24, (3), pp. 1045-1053, 2009.

[4] A. Gonzalez et al, "A sensitivities computation method for reconfiguration of radial networks," IEEE Trans. Power Syst., vol. 27, (3), pp. 1294-1301, 2012.

[5] L. Tang, F. Yang and J. Ma, "A survey on distribution system feeder reconfiguration: Objectives and solutions," in 2014 IEEE Innovative Smart Grid Technologies-Asia (ISGT ASIA), 2014, pp. 62-67.

[6] A. Borghetti, "A mixed-integer linear programming approach for the computation of the minimum-losses radial configuration of electrical distribution networks," IEEE Trans. Power Syst., vol. 27, (3), pp. 1264-1273, 2012.

[7] R. A. Jabr, R. Singh and B. C. Pal, "Minimum loss network reconfiguration using mixed-integer convex programming," IEEE Trans. Power Syst., vol. 27, (2), pp. 1106-1115, 2012.
[8] M. M. A. Abdelaziz, H. E. Farag and E. F. ElSaadany, "Optimum reconfiguration of droop-controlled islanded microgrids," IEEE Trans. Power Syst., vol. 31, (3), pp. 2144-2153, 2015.

[9] S. Chen, W. Hu and Z. Chen, "Comprehensive cost minimization in distribution networks using segmentedtime feeder reconfiguration and reactive power control of distributed generators," IEEE Trans. Power Syst., vol. 31, (2), pp. 983-993, 2015.

[10] Y. Wu et al, "Study of reconfiguration for the distribution system with distributed generators," IEEE Trans. Power Del., vol. 25, (3), pp. 1678-1685, 2010.

[11] H. Khodr et al, "Distribution systems reconfiguration based on OPF using Benders decomposition," IEEE Trans. Power Del., vol. 24, (4), pp. 2166-2176, 2009.

[12] J. A. Taylor and F. S. Hover, "Convex models of distribution system reconfiguration," IEEE Trans. Power Syst., vol. 27, (3), pp. 1407-1413, 2012.

[13] E. Dall'Anese and G. B. Giannakis, "Convex distribution system reconfiguration using group sparsity," in 2013 IEEE Power \& Energy Society General Meeting, 2013, pp. $1-5$.

[14] H. L. Hijazi and S. Thiébaux, "Optimal AC distribution systems reconfiguration," in 2014 Power Systems Computation Conference, 2014, pp. 1-7.

[15] Q. Peng, Y. Tang and S. H. Low, "Feeder reconfiguration in distribution networks based on convex relaxation of OPF," IEEE Trans. Power Syst., vol. 30, (4), pp. 1793-1804, 2014.

[16] J. Mendoza et al, "Minimal loss reconfiguration using genetic algorithms with restricted population and addressed operators: real application," IEEE Trans. Power Syst., vol. 21, (2), pp. 948-954, 2006.

[17] N. Gupta et al, "Multi-objective reconfiguration of distribution systems using adaptive genetic algorithm in fuzzy framework," IET Generation, Transmission \& Distribution, vol. 4, (12), pp. 1288-1298, 2010.

[18] M. Lavorato et al, "Imposing radiality constraints in distribution system optimization problems," IEEE Trans. Power Syst., vol. 27, (1), pp. 172-180, 2011.

[19] A. Zidan, M. F. Shaaban and E. F. El-Saadany, "Long-term multi-objective distribution network planning by DG allocation and feeders' reconfiguration," Electr. Power Syst. Res., vol. 105, pp. 95-104, 2013.

[20] I. Std, "60364-5-52,“," LV Electrical InstallationsSelection and Erection of Electrical Equipment-Wiring Systems," IEC, 2009. 